research workers in the field of ionospheric physics, as well as postgraduate students with a good grounding in basic physics.

In this new edition the presentation and the translation are substantially improved upon the old text. After reviewing the fundamental theory of electromagnetic wave propagation in a homogeneous isotropic and in a homogeneous magnetoactive plasma, the author treats propagations in inhomogeneous isotropic and inhomogeneous magnetoactive plasmas, making ample use of the methods of geometrical optics, and discussing at some length the limits of their applicability. Reflexion of radio waves from ionospheric layers is analysed in great detail. Radio wave propagation under cosmic conditions is discussed, including propagation in the solar coronal plasma and in the interstellar medium; a number of useful synoptic tables are given. The last chapter is devoted to non-linear phenomena occurring in a plasma placed in a variable electromagnetic field.

Throughout the whole book new material has been added and brought up to date. In particular, a number of non-linear phenomena have been presented in greater detail, and the discussion of pulse propagation and spatial dispersion has been greatly enlarged.

The book coverage, however, seems somewhat uneven, and a more expanded treatment would be desirable in some areas. Some topics of current interest to the magnetosphere physicist, such as wave-particle interactions, instabilities and wave generation associated with particular plasma distributions in velocity space, beam-plasma instabilities, and so on are mentioned very briefly or even totally omitted.

This apparent shortcoming may be partially due to the fact that the original Russian text of this second edition was published as long ago as 1967. This reflects in the bibliographic section, where almost 1,200 references are given, covering mainly papers up to only 1966 . Of these references 383 are actually referred to in the text, while the others are intended, according to the author, to help in familiarizing the reader with recently published work. They span a period from 1958 to 1967 . A minor drawback of this book, when used as a reference work, lies in the difficulty of locating some of the symbols in a non-alphabetical list given over three pages at the beginning of the text.

Like the first edition, this new edition maintains the highest standard of scholarship and exposition and, within the limitations discussed above, the author has unquestionably achieved the objective of producing a comprehensive and authoritative work.

G. Martelli

\section{Arithmetic of Evolution}

An Introduction to Population Genetics Theory. By James F. Crow and Motoo Kimura. Pp. xiv +591 . (Harper and Row : New York and London, November 1970.) £6.05.

THE authors have been far too modest in describing this book as an "Introduction" to the theory of population genetics. They have given us a thorough review of the present state of the mathematical theory of population genetics, with references to work published as recently as 1969 .

The reader will need to be familiar with genetical terms and ideas. Apart from a useful appendix on statistical and mathematical methods, few concessions are made to the reader unfamiliar with the mathematical formulation and manipulation of problems and with the interpretation of mathematical solutions. Inevitably, the mathematics is sometimes fairly advanced but numerical results and graphs are used to illustrate the conclusions. There are problems at the end of most chapters, and there is also an excellent bibliography.

The emphasis is almost entirely on evolutionary processes and the theory of natural selection. There is very little on the theory of the imposed type of selection used in animal and plant breeding programmes. The authors have deliberately avoided lengthening the book by omitting discussion of the experimental and observational data that have been used to develop and verify the theory. This decision is inevitable but sometimes creates difficulty in assessing the relative importance of different topics and in understanding exactly what it is that has to be explained or predicted.

On the technical side, I would like to have seen more use made of Markov chains and transition matrices in the discussion of finite populations and to have had some comments on the degree of relevance of results about asymptotic equilibria and stable distributions that are based on the concept of an environment constant over an infinite number of generations.

This book clearly meets the need for an advanced text. We still await more elementary texts to be used at undergraduate level for both mathematicians and biologists. Hopefully, one of these texts will emphasize breeding and selection programmes and hence the theory of artificial selection as well as natural selection.

Altogether this is an excellent book, finely produced, and is likely to be a standard work for the specialist teacher, research worker or graduate student.

R. N. Curnow

\section{Embryology in vitro}

Organ Culture. Edited by J. Andre Thomas. Pp. xiii +512. (Academic: New York and London, September 1970.) £13.75.

THE present status of organ culture owes much to Etienne Wolff and his school, and this well presented book is essentially a showcase for their efforts. It is a translation of Cultures Organotypiques published in France in 1964, and the several authors, all colleagues of Wolff's, have taken the opportunity to update their reviews to 1969 by means of an addendum to each chapter.

After an opening discussion by Wolff on the general principles of organ culture, there are nine chapters concerning different areas of research utilizing these techniques. Differentiation of embryonic organs is the principal theme, and the clear message is that this usually occurs autonomously in accordance with normal development, whether cultured in natural or synthetic media. Within this general framework, consideration is given to interactions of cells and of tissues, the effects of hormones and inhibitors, and the culture of malignant tumours and various invertebrate tissues. There is almost no discussion on the culture of adult organs for, in Wolff's words, "These can be made to survive by means of aseptic perfusion techniques."

Not unnaturally, most of the authors are at pains to point out the value of their studies, but with the result that the text sometimes reads like a catalogue of experiments. Although styles vary, one or two chapters present a lot of information as terse stattments of apparent fact, and one can only hope that these are really critical selections from the extensive literature. The addenda are. obviously an easy way of updating the original articles, but there are occasions when the later information either simply confirms the previous reports or renders them redundant, except for historical interest. On the whole, however, this is an interesting and worthwhile collection.

The translators have done a good job. The text generally reads well, errors are relatively scarce, and they have even noted discrepancies in the magnification figures for some plates. The price of the book is high enough to put it beyond the reach of many aspiring, and even established, embryologists. This is a pity, for it illustrates well not only the highways and by-ways of a profitable method of research, but also the development of one man's influence on his science. We have come a long way since 1859 when the French anatomist Vulpian removed large fragments of tissue from frog embryos and "cultured" them in ordinary water. W. D. Billington 